\title{
Mobile Charging as a Service: A Reservation-Based Approach
}

\author{
Xu Zhang, Yue Cao, IEEE Member, Linyu Peng, Jichun Li, Naveed Ahmad and Shengping Yu
}

\begin{abstract}
This paper aims to design an intelligent mobile charging control mechanism for Electric Vehicles (EVs), by promoting charging reservations (including service start time, expected charging time, and charging location, etc.). EV mobile charging could be implemented as an alternative recharging solution, wherein charge replenishment is provided by economically mobile plug-in chargers, capable of providing on-site charging services. With intelligent charging management, readily available mobile chargers are predictable and could be efficiently scheduled towards EVs with charging demand, based on updated context collected from across the charging network. The context can include critical information relating to charging sessions as well as charging demand, etc. Further with reservations introduced, accurate estimations on charging demand for a future moment are achievable, and correspondingly, optimal mobile chargersselection can be obtained. Therefore, charging demands across the network can be efficiently and effectively satisfied, with the support of intelligent system-level decisions. In order to evaluate critical performance attributes, we further carry out extensive simulation experiments with practical concerns to verify our insights observed from the theoretical analysis. Results show great performance gains by promoting the reservation-based mobile charger-selection, especially for mobile chargers equipped with suffice power capacity.
\end{abstract}

Note to Practitioners-The convenience of charging service is one major concern for EVs, especially when an urgent charging is required while none charging points are reachable. Recently, a Chinese EV company (NIO) is promoting its mobile charger (ES8 model) to Tesla. Driven by such market trend, this paper proposes an efficient approach toward intelligent scheduling of mobile chargers toward parked EVs. Different from fixed charging stations focusing on the problem of long waiting times, the proposed solution is applicable to chargingon-demand with pre-charging appointment at mobile chargers. Preliminary experiments show great charging efficiency achieved by concerning the issue of where to reserve, i.e., the consideration of optimal selection on mobile chargers. Such mobile charging services can coexist with the governmental or pilots' initiated charging station deployment. However, future research will need to evaluate the holistic service platform.

Index Terms-Electric vehicles (EVs), battery charging, queuing theorem, mobile charging services.

$\mathrm{Xu}$ Zhang is with the Department of Computer Science and Engineering, Xi'an University of Technology, Xi'an, China, e-mail: zhangxu@xaut.edu.cn

Yue Cao (corresponding author) is with the School of Transportation Science and Engineering, Beihang University, China, e-mail: $871441562 @$ qq.com

Linyu Peng is with the Department of Mechanical Engineering, Keio University, Japan, e-mail: 1.peng@mech.keio.ac.jp

Jichun Li is with the School of Computer Science and Electronic Engineering, University of Essex, UK. e-mail: j120340@essex.ac.uk

N.Ahmad is with the Department of Computer Science, University of Peshawar, Pakistan, e-mail: n.ahmad@upesh.edu.pk

S.Yu is with the State Key Laboratory of Synthetical Automation for Process Industries, Northeastern University, China, e-mail: spyu @ mail.neu.ed.cn

The work is supported in part by the National Natural Science Foundation of China (U1864206).

\section{INTRODUCTION}

W ITH growing concerns on economic as well as environmental issues, there have been considerable amount of efforts on sustainable energy development. Vehicles of electricity propulsion-based such as Electric Vehicles (EVs) are one key enabler for the evolution of sustainable transport technology. As compared to traditional internal combustion engine (ICE) vehicles powered by gasoline, EVs are gaining popularity from the general public mainly driven by its energy efficiency as well as low greenhouse gas emissions. The recent advances in EVs engineering have made them more performing and attracted more road users. Forecasts are expecting at least 250 million EVs to hit the road globally by 2030, and predicting more EVs than gasoline-powered cars within two decades [1].

Even though, EVs still require a significant amount of time to replenish their energy, and the frequency for charging services is relatively high. It typically takes hours to fully charge a depleted EV battery [2], compared to only a few minutes for a gas-powered vehicle. A Nissan Leaf EV on a full charge can achieve up to 107 miles of range [3], which still may not be enough to cover additional driving. The limitations in battery capacity have been considered as one of the major barriers to large-scale adoption of EVs, commonly known as range anxiety. While there are solutions to alleviate such concerns, such as quick chargers (i.e., level-3 chargers) and battery swapping techniques [4], big capital investment decisions have to be made. At areas with low EV adoption rates, it is even hard to profit from building costly fast charging (or swapping) stations [5].

Additionally, due to the mobility in nature, randomly distributed EVs have various charging needs that vary both temporarily and spatially [16][21]. The state-of-charge (SOC) with individual EVs changes over time, and EVs with charging requirements may locate at places where public fixed charging stations (FCS) are few and far between. As of yet, an EV with low-SOC has to make certain detours towards a public staticlocation charging station, where the EV could be left stranded by overcrowding, due to limited charging outlets (charging capacity) with the station. Inevitably, the travel efficiency as well as driving comfort could be degraded. The major obstacles to resolve the above range and charging issues are the lack of feasible charging services.

In comparison, EV mobile charging is believed to be economical as well as feasible [23][28], mainly due to its easy deployment in nature, e.g., by taking the form of a conventional van with plug-in chargers on-board. Compared to 
conventional fixed charging mode, mobile charging facilitates services by instantly and proactively responding to demand especially at times of potential grid stress. Based on certain mobile charging management, the range anxiety could be significantly alleviated. Within this realm, one challenging issue is how to select the most appropriate mobile charger to achieve assured service experiences for EVs, with practical concerns including limitations on the amount of mobile chargers and their charging capacity. Further, the mobility nature with individual mobile chargers contributes to the challenge as well. As of yet, most research works target the static charging mode, and limited efforts have been put into the concept of mobile charging. Besides, no restrictions are applied with existing relevant works on mobile charging service [23], where chargers (and EVs) are supposed as stationary loads. However, such assumptions does not conform to the real case in practice [28].

In this paper, we focus on the design of an intelligent mobile charging platform with more practical concerns, i.e., limited number of mobile chargers equipped with restricted charge capacity, wherein a recharging may need when the charger's battery SOC is low. As such, the availability regarding mobile chargers is one key concern, which is our focus in this work. With the support of charging reservations (including service start time, expected charging time, and charging location, etc.), accurate estimation on key indicators for available mobile chargers becomes possible. Correspondingly, charging demands across the network can be more efficiently and effectively satisfied.

Toward this end, we propose a reservation-based mobile charging scheduling scheme, aiming to provide efficient as well as effective EV charging services of system-level. By intelligently scheduling optimal mobile chargers toward vehicles with appointments, great feasibility can be achieved for both service providers and EVs.

The technical contributions from this paper can be summarized as follows.

1) An intelligent mobile charging framework is proposed, with practicality concerns taken into account. By accounting for concerns including limitations on chargers, and spatiotemporal dynamic variations regarding chargers as wellas charging demands, efficient demand-response mobile charging services across the whole network can be enabled.

2) Over the proposed mechanism, reservations on mobile charging services are introduced, whereby accurate estimations on available mobile chargers can be achieved for a future moment, thereby enabling optimal charging management to timely meet demand of EVs. Particularly, charging reservations are associated with each charger, while the reservation-allocation among all chargers contributes to the major challenge.

This paper is organized as follows: Related work is briefly reviewed in Section II. We then present the performance model and conduct theoretical analysis in Section III. In Section IV, we elucidate our proposed intelligent mobile charging scheduling scheme. The performance of the proposed framework is evaluated in Section V through extensive simulations and we conclude the paper in Section VI.

\section{RELATED WORKS}

More types of EVs are emerging and many have already been released to markets. Apart from pure EVs [2][3], plugin hybrid EVs (PHEVs) [29] combine characteristic of a conventional ICE system with electric motor, which allow for alternative energy power when the battery is depleted. And yet, charging is a major concern on the wide adoption of EVs [1]. Many works have proposed to incentivize EVs not to charge at locations or during periods of high demand [6] (e.g., to avoid peaks (peak demand hours)). The Society of Automotive Engineers (SAE) have defined the communication standards when an EV is being charged [12][15].

\section{A. Fixed Plug-in Charging Systems}

Implemented as permanent stations connected to the power grid, FCSs provides EVs energy replenishment at static charging sites where multiple charging facilities are installed. EV users need to drive toward a specific FCS for battery recharging during their journeys. Key considerations with FCSs are generally of two-aspects: the installation phase and the operational stage.

With FCSs deployment, there is mainly constructive work, including the site selection intended for the installation, along with the number of FCSs to be established. During this process, the deployment can be quite costly relating to labor and material expenses [13]. There are also studies looking at the optimal number of FCSs to be deployed [14].

As for FCS operational aspect, the major concern is the fact that there are too many EVs with charging demand, compared to insufficient FCSs installed [21]. As a result, FCSs can quickly become saturated crowded with EVs. Therefore, many research works have emerged to work on such issue, in order to optimally schedule EVs for high station utilization. Most existing researches mainly focus on the issue of where to charge [17][19][20][22][27]. An optimal FCS is selected with charging decision making [7][8][18], through constantly interaction between EVs and other participants, such as FCSs. Game theoretic models are extensively employed for modelling charging interactions [9][10]. Also, optimal pricing is achieved through maximization of individual utility functions through Nash equilibrium evaluations [11].

Regardless of the efforts put forth into charging scheduling, driver etiquette directly affects the true utilization of charging stations. Once the EV is fully charged, people often simply forget to move the vehicle, which intensifies the imbalance between supply and demand.

\section{B. Mobile Charging}

A potential solution to maintain charging efficiency is the EV mobile charging paradigm [23][28]. The design of a mobile charger can take the form of a conventional van with plug-in charging facilities, e.g., the FreeWire mobi charger [28]. Instead of plugging into a static station, drivers can park wherever they like. The great feature of the mobile charging design is the capability to bring charging services to EV side, thereby enabling flexible as well as effective charging services. 
As an alternative, the mobile charging techniques offer great feasibility to alleviate hassle with public CS. It resolves the permanent station space problem, by orchestrating mobile chargers moving around. Besides, big capital investment can be saved by eliminating massive FCSs constructions.

\section{Motivation}

As of yet, there have been limited research efforts to consider the charging control with regard to mobile charging scenarios [23]. A good understanding is lacked on mobile charging scheduling control. In this paper, we focus on such issue and aim to provide an intelligent scheduling mechanism for achieving efficient mobile charging. By characterizing critical charging behaviors with both mobile chargers as well as EVs, we are able to obtain accurate estimation on critical performance indicators, such as available times regarding specific mobile charging unit. In particular, we develop a reservation-based mode to allow for advanced booking for future charging demand. Over literature, this work develops a viable mobile charging framework empowered by reservation feature, which can work with practical concerns on network dynamic patterns in large scale scenario.

With the support of the mobile charging intelligence, EV users are able to book an optimal mobile charger and receive services at the reservation time. From system-level view, higher utilization of power grid resources could be achieved. And such central controller generally has sufficient network information as well as high computation power, charging demand could be more efficiently distributed.

\section{System ModeL}

This section introduces the intelligent mobile charging framework, and a simple analytical modelling is formulated to derive a closed-form of the concerned waiting times of EVs.

\section{A. Overview}

We consider a city scenario where FCSs are geographically deployed, and a couple of mobile power units (MPU) (or mobile chargers) are owned and managed by individual FCSs, as shown in Fig. 1. The MPU could take the form of a conventional van with plug-in batteries on-board, capable of providing a level-2 (or even level-3) charging service. MPUs could be powered by gasoline, with their electric power packs associated with SOC thresholds. As such, a limit value regarding MPU-SOC can be set so as each mobile charger can make decision on when to get energy replenishment. A central aggregator (CA) globally manages the mobile charging process for all EVs in the network.

As illustrated in Fig. 1, an EV needs additional charging while on-the-move or parked. A mobile charging service is then demanded by the EV and sent to the CA, along with a range of charging context information. Such context could include the parking location, desired serving time point and charging duration, etc. In the case that a mobile charging request is made while on the move, the EV will then drive toward an appropriate parking spot. Upon receiving the demand, the CA makes enquires to all FCSs for candidate

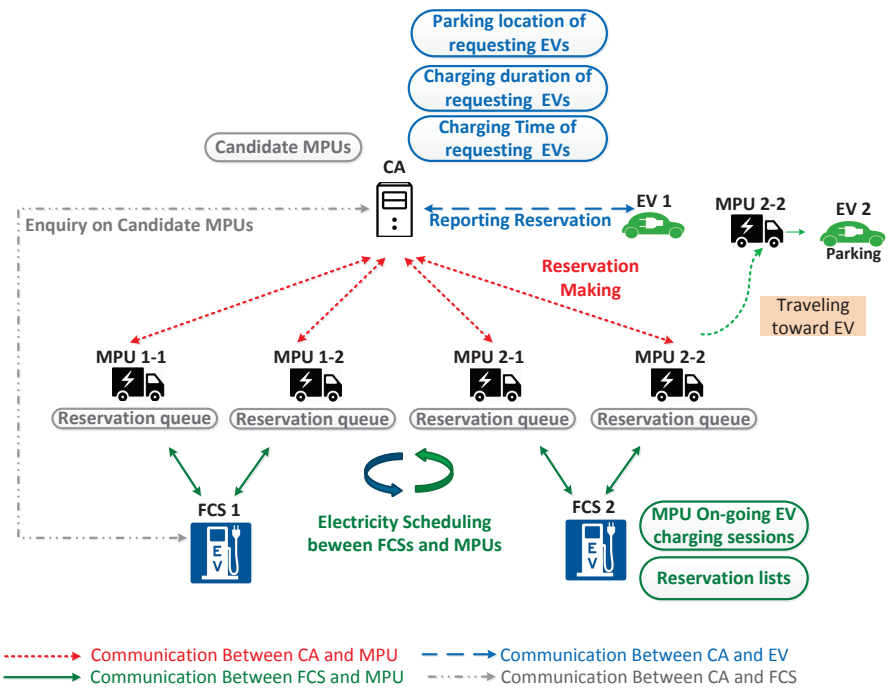

Fig. 1. An illustration of an intelligent EV mobile charging paradigm

MPUs ${ }^{1}$. By computing locally, each FCS replies a candidate MPU to the CA. The CA then selects an optimal MPU from the candidates based on a global MPU-selection decision making, and notifies the requestor EV of the best MPU choice. Upon receiving the MPU arrangement, the EV then reports its charging reservation to the CA at the MPU. As such, a reservation queue will be formed at the candidate MPU with the help of CA orchestration. Under our scenario, each MPU is assumed to be able to charge one EV at a time, which is in line with practical scenarios [25].

- Communication Requirement: Generally, EVs are equipped with wireless devices (e.g., 3G/LTE, etc. [22]), that allow for communicating with the CA via cellular networks, facilitating reservation-making for future charging demand.

Specifically, the communication between CA and MPUs will be enabled, so as to make reservations for EVs and allow for tracking MPU locations. In addition, MPUs will contact the CA for choosing appropriate FCSs to replenish energies when their SOCs are low. FCSs will also communicate with the CA in reply to candidate MPUs enquires. And all communications will be supported by ubiquitous cellular networks.

Particularly, due to limited number of MPUs against heavy charging demand from EVs in the network, one key issue is to estimate the availability regarding individual MPUs. Here, we advocate to resolve such issue by promoting charging reservations as discussed above. Toward this end, available times relating to MPUs will be approximated at FCS side, mainly owing to the feasibility for the MPU owner (i.e., FCSs) to monitor on-going charging sessions as well as reservations with MPUs [28]. Globally, the CA receives candidate MPUs from all FCSs in the charging network, and making decisions on optimal MPU-selection. Additionally, once an MPU needs electric energy replenishment (in terms of a low MPU-SOC

\footnotetext{
${ }^{1}$ Such enquiry is sent to FCS in accordance with practice that MPUs are generally owned and managed by FCSs. However, the proposed scheme also applies to the case that the CA directly contacts MPUs.
} 
TABLE I

LIST OF NOTATIONS

\begin{tabular}{ll} 
Symbol & Description \\
\hline$S O C_{c u r}$ & presently available energy of the EV battery in terms of \\
& SOC \\
$S O C_{\max }$ & fully-charged EV battery of SOC \\
$T$ & EV parking-tolerance threshold \\
$T_{w}$ & reservation queueing time (or waiting time) \\
$T_{t r a}$ & travel time for the selected MPU to take from its current \\
& location to the EV parking spot \\
$t_{e v}^{r e s}$ & time slot reserved for recharging the EV battery \\
$t_{c u r}$ & current time in network \\
$t_{e v}^{\text {pur }}$ & EV's parking time \\
$t_{i d l e}$ & earliest available charging time slot \\
$\delta_{e v}^{c h a}$ & expected charging duration of the EV battery \\
$E$ (Joules) & the electricity capacity of the EV battery \\
$r_{e v}^{-}(\mathrm{J} / \mathrm{meter})$ & EV energy consumption rate \\
$r_{m c}^{+}($Watts $)$ & mobile charger charging rate \\
$r_{M P U}$ & MPU travelling speed \\
$d$ & EV traveling distance from current location to its destination \\
$n_{c}$ & \# of EVs under charging \\
$n_{r}$ & \# of EVs reserved for charging \\
$c$ & charging capacity of a FCS, i.e., \# of mobile chargers \\
$N_{F C S}$ & \# of FCSs \\
\hline &
\end{tabular}

value), the CA will orchestrate the MPU towards a spatially closest FCS for a refill. In such cases, any on-going charging sessions with the MPUs would be terminated.

In reality, the CA is believed to be equipped with a super power and super computation capability to make charging plans for all EVs, allowing for demand-response functionality across a large scale network. Such intelligent mobile charging service enables to provide high charging efficiency especially with concerns on the perspective of high EVs demand.

\section{B. Analytical Modelling}

Typically, a FCS owns a set of $c$ mobile chargers (MPU), each of which has a charging rate of $r_{m c}^{+}$. An EV is equipped with a battery of capacity of $E$, and consumes the energy at the rate of $r_{e v}^{-}$. A list of variable notations are given in Table I. We start our modelling from a single fixed charging station, and as such the proposed queueing model quite resembles a classical $M / M / c$ queueing system with the following assumptions.

- $\lambda_{s}, \mu_{s}$ for any charging station: The charging requests are commonly assumed to arrive according to a Poisson process, i.e., exponential interarrival times of requests with mean $1 / \lambda_{s}$; exponential charging time with mean $1 / \mu_{s}$.

- Service Discipline: Requesting EVs are served by following the order of their reservation times, i.e., EV with the earliest-reservation-time is allocated with the highest charging priority.

The above assumptions allow for analysis on mobile charging process and captures reality in many cases, especially in a dense service charging scenario [23]. Generally, for a large pool of potential EV users, the probability for individual EVs to request charging is independent and thus, a Poisson process is typically adopted [24].

Therefore, the model can be characterized by the number of EV customers in the system $\{0,1, \ldots, c, c+1, \ldots\}$.
Based on the stochastic process theory [30], we thus denote $p_{n}$ as the stationary probability that there are $n, n \in$ $\{0,1, \ldots, c, c+1, \ldots\}$ customers in the system, thereby vector $\mathbf{P}=\left(p_{n} \mid n=0,1, \ldots, c, c+1, \ldots\right)$. Along with the generator matrix $\mathbf{Q}$ (which can be easily obtained according to the $M / M / c$ queuing metrics), the steady state probabilities $\mathbf{P}=\left(p_{n} \mid n=0,1, \ldots, c, c+1, \ldots\right)$ can thus be derived by solving the matrix equation $\mathbf{0}=\mathbf{P} \cdot \mathbf{Q}$.

Next, we use the established analytical framework above to mathematically derive critical design parameters that relates to the efficiency of the mobile charging system, with regard to key indicators such as, service delay, etc.

1) MPU Occupied Probability: An important quantity is the probability that all of the $c$ MPUs are fully occupied, which implies that EVs have to wait before charging. Denote this probability by $\Pi$, which refers to as the MPU occupied probability. By PASTA the probability could be derived as the Erlang C formula

$$
\begin{aligned}
\boldsymbol{\Pi} & =\sum_{n \in\{c, c+1, \ldots\}} p_{n} \\
& =\frac{\left(c \rho_{s}\right)^{c}}{c !}\left(\left(1-\rho_{s}\right) \sum_{k=0}^{c-1} \frac{\left(c \rho_{s}\right)^{k}}{k !}+\frac{\left(c \rho_{s}\right)^{c}}{c !}\right)^{-1}
\end{aligned}
$$

Where $\rho_{s}=\lambda_{s} /\left(c \mu_{s}\right), \rho_{s}<1$, indicating the charging demand intensity. Clearly, a higher value of $\rho_{s}$ indicates a heavier demand intensity case.

Note the value of $\Pi$ is mainly governed by two parts, the on-going charging queue (i.e., $\left\{p_{n} \mid n=c\right\}$ ) and reservations queue (i.e., $\left\{p_{n} \mid n \geq c+1\right\}$ ), wherein the charging demand $(n \geq c+1)$ beyond the charging capacity $(c)$ has to reserve for charging service.

2) Mean Service Delay: The mean service delay for a requesting $\mathrm{EV}$ is denoted as the time duration from the reserved charging time to the arrival of the MPU. The delay is mainly composed of two parts: the expected queueing time for available MPUs, denoted as $E\left(W_{q}\right)$, and the mean driving time of MPUs to requesting EVs, referred to as $E\left(W_{d}\right)$. We thus have the mean service delay denoted by

$$
E(W)=E\left(W_{q}\right)+E\left(W_{d}\right)
$$

From Little's law, we directly obtain the expected queueing time for an available MPU

$$
E\left(W_{q}\right)=\Pi \cdot \frac{1}{1-\rho_{s}} \cdot \frac{1}{c \mu_{s}}
$$

In order to compute $E\left(W_{d}\right)$, we first consider a simple case of a unit square area of $[0,1]^{2}$. Let $\left(X_{i}, Y_{i}\right)(i=1,2)$ be i.i.d. variables that have uniform distribution on $[0,1]^{2}$. According to [26], the exact PDF for $\left|X_{1}-X_{2}\right|$ is given by

$$
f_{X}(x)= \begin{cases}2(1-x) & 0 \leq x \leq 1 \\ 0 & \text { otherwise }\end{cases}
$$

Hence, the average distance $d$ between two random locations in a unit square area is derived as

$$
d=\iint_{x, y} f_{X}(x) f_{Y}(y) \sqrt{x^{2}+y^{2}} d x d y
$$




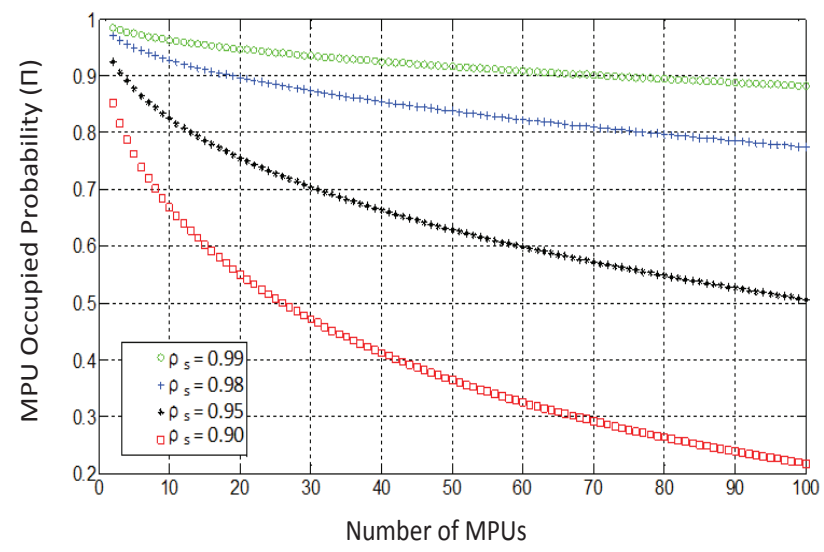

Fig. 2. MPU occupied probability (П) with the variance of $\rho_{s}$

And if we extend the result of Eq. (5) to an area of $[0, a]^{2}$, the average distance approximately equals to $a \cdot d$. Therefore, for a square area of $[0, a]^{2}$ along with a constant MPU moving speed $\left(r_{M P U}\right)$, the mean driving time $E\left(W_{d}\right)$ of MPUs to requesting EVs could be further refined to

$$
E\left(W_{d}\right) \approx \frac{a \cdot d}{r_{M P U}}
$$

Next, we further extend our single FCS modelling to a network of multi-FCSs. Consider a number of $m$ FCSs, wherein each has a set of $c_{i}(i=1,, m)$ MPUs. The model is thus a parallel of a set of $M / M / c_{i}$ queues. Similarly, the multi-FCSs model could also be characterized by giving a transition matrix denoted by

$$
\mathbf{Q}_{\mathbf{M}}=\left(\begin{array}{lll}
Q_{1} & & \\
& \ddots & \\
& & Q_{m}
\end{array}\right)
$$

Wherein $Q_{i}(i=1, \ldots, m)$ refers to a block of matrix. We thus denote $\mathbf{P}_{i}$ as the stationary distribution vector of a single FCS model, thereby vector $\mathbf{P}_{\mathbf{M}}=\left(\mathbf{P}_{i} \mid i=1, \ldots, m\right)$ representing stationary distributions for a multi-FCS case. By solving $\mathbf{0}=\mathbf{P}_{\mathbf{M}} \cdot \mathbf{Q}_{\mathbf{M}}$ according to [30], each element $\mathbf{P}_{i}$ can thus be derived.

Therefore, by computing the mean service delay values $\left\{E_{i}(W) \mid i=1, \ldots, m\right\}$ of individual FCSs based on Eq. (2), a minimal value $E_{\min }(W)$ regarding the shortest mean service delay would be obtained

$$
E_{\min }(W)=\min \left\{E_{i}(W) \mid i=1, \ldots, m\right\}
$$

As will be evaluated in later sections, the value of $E_{\min }(W)$ corresponds to an important context concerned for intelligent MPU-selection decision making.

3) Numerical Results: We show in Fig. 2 and 3 the MPU occupied probability and the mean service delay under varied intensity $\rho$, with $\mu$ fixed. The mean driving time $E\left(W_{d}\right)$ is set as a constant value as given in Eq. (6) for simplicity, and such restriction will be relaxed in our simulation experiments. As observed, both performance metrics are reduced with a larger $c$, indicating that desirable performance gains will be achieved when more MPUs deployed.

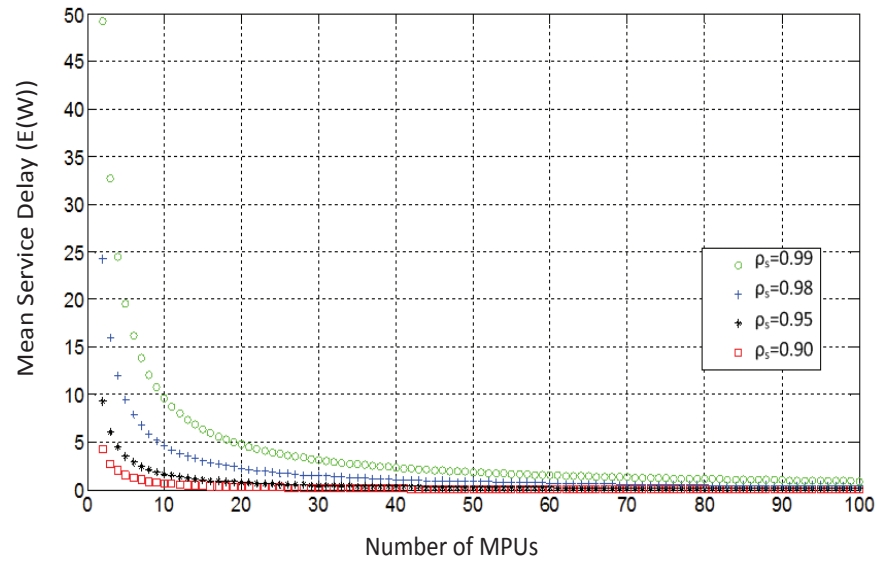

Fig. 3. Mean service delay $(E(W))$ with the variance of $\rho_{s}$

In Fig. 2, we can see that when the load at the FCS becomes heavier (higher value of $\rho$ ), the MPU occupied probability increases significantly. Specifically, for a high service demand intensity $(\rho=0.99)$, a requesting EV could be delayed to receive its charging service at a probability of above 0.9 , with the FCS holding the number of MPUs less than $c=70$.

Fig. 3 shows that the mean service delay decreases with the increment of $c$, especially for a small deployment of MPUs (less than 10). It is interesting to show that with more MPUs deployed, the performance gains are not improved much. Such analysis will be further proved in our latter simulation evaluations.

With observations from Fig. 2 and 3, we can see that a large deployment of MPUs could enhance the overall performance gains at a FCS. However, the improvement is not significant. Such an observation could have vital practical meaning, especially when large amount of MPUs taking up too much space and the control becomes complex. On the other hand, the upgrade on charging power could be an attracting option for achieving significantly enhanced system performance, which will be analyzed latter in our experiments.

The modelling provides an analytical view for the performance characteristics. To further account for more realistic designing parameters, such as the parking threshold of a requesting $\mathrm{EV}$, a protocol is presented in the following section.

\section{The Proposed Scheme: Reservation-Based Mobile Charging SCHEDULING}

Considering the high dynamics of EV charging demands, with respect to varied SOCs as well as reservation heterogeneity (i.e., various reservation times and locations), it is vital to provide real-time monitoring of charging conditions across the network. Therefore, we propose a centralized scheme to support efficient mobile charging services of system-level decisions.

\section{A. Protocol for Mobile Charging Scheduling}

Consider an EV, i.e., $e v_{r}$, that requires additional charging while it is parked. The vehicle realizes such charging demand (either moving or parked) and then interacts with the orchestrator CA directly for desired MPU selection, such as less 


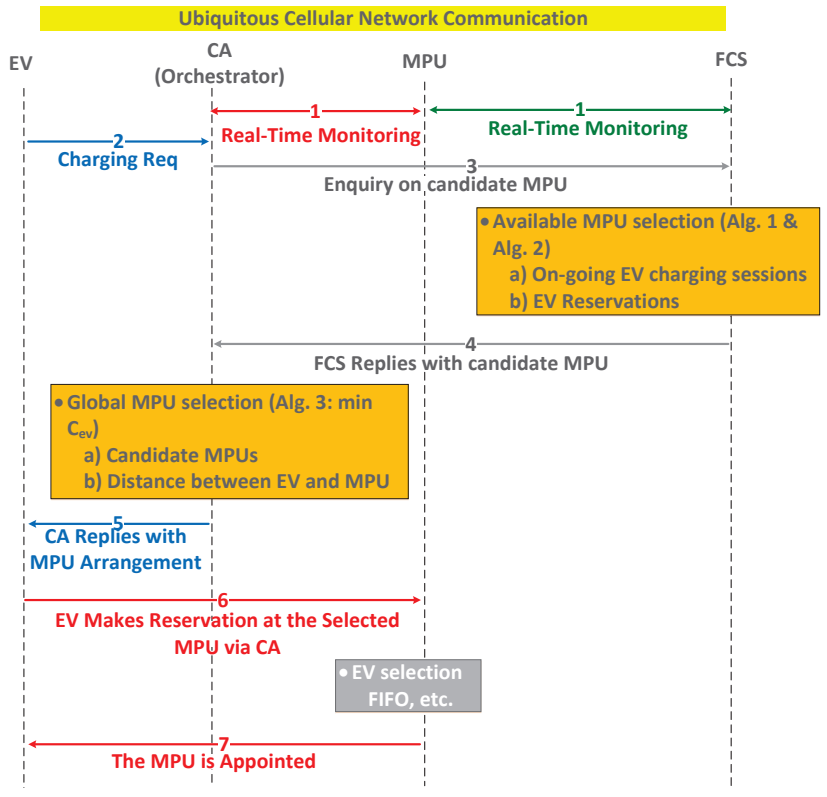

Fig. 4. Intelligent mobile charging sequence chart

waiting time, etc. The typical procedure is stated as follows, which is illustrated in Fig. 4.

Step 1: Real-time monitoring: Periodically, the CA keeps track on all MPUs for positioning locations. Individual FCSs also monitor their owned MPUs charging status, such as ongoing charging sessions and reservations.

Once the MPU realizes its loaded electric power pack not enough for an upcoming EV charging demand (e.g., measured by MPU-SOC value), it will drive toward a nearest FCS for energy replenishment. For simplicity and to focus on EV charging schedulling, we assume the MPU can access the charging point directly once arrived at the FCS without waiting.

Step 2: Reporting charging demand: When the $e v_{r}$ needs recharging, it makes an enquiry to the orchestrator CA for desirable MPU options.

Step 3: Enquiry on candidate MPUs: The CA makes enquires to all FCSs in the network for available MPU lists.

Step 4: Available MPU selection: Upon receiving the request from the CA, each FCS locally executes the available MPU selection process (based on Alg. 1 and Alg. 2 as will be presented in latter section), which determines the candidate MPU with concerns on on-going EV charging sessions and charging reservations of individual MPUs. Notice that each FCS is capable to calculate a candidate MPU locally, so that the CA is able to save massive communication efforts with each MPU, especially when the mobile chargers in large number.

Step 5: Global MPU selection: By intelligently compiling a list of candidate MPUs reported from all FCSs, the CA then replies to the query EV with a best choice of an MPU (according to Alg. 3 as will be stated in latter section). Such intelligence is supported by the operation of the Global MPU Selection procedure, by taking into account charging waiting times, charging duration as well as travel distances.

In a steady system, the majority of MPUs will mostly be on- the-move, their locations are also important context factors for MPU-selection decision making, so as to enable estimations of real-time distances between MPUs and requesting EVs.

Step 6: Reporting reservations: Notified by the CA of the MPU-selection decision, the requestor $e v_{r}$ then informs the CA by sending a reservation request message to the recommended MPU, which may contain the following fields (variables defined in Table I):

- Identification of the EV

- Presently available energy of the EV battery $\left(S O C_{c u r}\right)$

- Fully-charged EV battery level $\left(S O C_{\max }\right)$

- Energy consumption rate of the vehicle $\left(r_{e v}\right)$, which equals 0 when the vehicle is parked

- The EV's parking location: Provided prevalent installation of on-board navigation systems at EVs, the exact parking spots of individual EVs are easy to known at the CA.

- Expected time to be served $\left(t_{e v}^{r e s}\right)$ : The time slot that an EV expects the MPU to recharge its battery.

- Expected charging time duration $\left(\delta_{e v}^{c h a}\right)$ : The expected time duration for charging an EV upon the arrival of a mobile power unit.

- Parking tolerance duration $(T)$. It is usually a variable that represents the anticipated parking period of the vehicle

Step 7: Service discipline (or EV selection): Once there is a reservation list formed at an MPU, the MPU performs the EV selection procedure based on the service discipline, which determines the next EV to be served. The discipline can include: the FIFO (select EV according to the order of their reservation times) and the distance (select EV that is in spatial proximity to the current location of an MPU), etc. An MPU is then appointed by the CA to the target EV.

Note that EV users tend to park for a limited amount of time, and we herein take into account the Parking duration concerns: An EV starts its tolerance threshold timer once it makes the charging reservation. And the charging process could be terminated anytime once the EVs parking duration hits/exceeds the threshold.

Particularly, the CA makes records of each reservation information, e.g., $<v_{i d}, m_{i d}, t_{e v}^{r e s}, \delta_{e v}^{c h a}>$, wherein $v_{i d}$ and $m_{i d}$ indicate the identifications of the vehicle and reserved MPU, respectively. And corresponding records are deleted once the requesting charging process is finished.

As noticed, there are two main functions involved to achieve the proposed mobile charging intelligence: the MPU scheduling procedure and the EV selection procedure. With the MPU scheduling, an expected charging cost value, denoted as $C_{e v}$, is examined for each candidate MPU regarding the requesting $\mathrm{EV}$, and the MPU with the minimal value is appointed, as formulated in Eq. (9). The value of $C_{e v}$ is mainly determined by three parts: the reservation-queueing time $\left(T_{w}\right)$ (or waiting time), the travel time $\left(T_{t r a}\right)$ for the selected MPU to take from its current location to the EV parking spot, and the expected charging time duration $\left(\delta_{e v}^{c h a}\right)$.

$\min C_{e v}=\min \left\{\left(T_{w}+T_{t r a}+\delta_{e v}^{c h a}\right) \mid\right.$ candidate $\left.M P U s\right\}$ 
Please be noted that the expected charging time duration $\left(\delta_{e v}^{c h a}\right)$ also considers the case when the requesting EV may continue to consume energy after reporting its reservation, since the EV may still be on-the-move before parking taking place. While the expected charging time $\left(\delta_{e v}^{c h a}\right)$ is easy to obtain, the challenge is with the determination of $T_{w}$. As discussed previously, the key problem here is to accurately estimate the available charging time at candidate MPUs prior to the current requesting EVs reserved time. The prediction to determine the value of $T_{w}$ will be presented in the following section. For EVs with parking threshold, the EVs will wait for the reserved mobile charging service until the parking deadline, and the charging session will be terminated once the parking deadline is reached.

\section{B. Charging Cost Estimation $\left(C_{e v}\right)$}

As discussed previously, in order to intelligently respond to charging demands, the CA needs to keep track of the expected charging cost value $\left(C_{e v}\right)$ associated with each candidate MPU for a future moment. One key determination factor is the expected waiting time $\left(T_{w}\right)$. To accurately approximate the value of $T_{w}$, not only on-going charging sessions, but also reservations need to be taken into account. Following our previous discussions, we thus define two kinds of EV queues: on-going charging session $\left(N_{c}=\left\{1, \ldots, n_{c}\right\}\right)$ and reservation queue $\left(N_{r}=\left\{1, \ldots, n_{r}\right\}\right)$, respectively. Estimations on each part based on Eq. (8) will be approximated as the following.

1) Estimating Expected Charging Time $\left(\delta_{e v}^{c h a}\right)$ : The EV may report its reservation while on-the-move and thus, additional energy has to be consumed. Therefore, the energy consumption consists of two parts: the current state and the future state. With SOC readily available at the $\mathrm{EV}$, it is easy to obtain the energy consumed already. To additionally account for the EV traveling distance of $d$ from current location to the parking destination, the consumption on the additional electricity can be computed as $d \cdot r_{e v}$ (Joules).

Therefore, the expected charging time duration can be formulated as the following.

$$
\delta_{e v}^{c h a}=\left(\left(S O C_{\max }-S O C_{c u r}\right) \cdot E+d \cdot r_{e v}\right) / r_{m c}
$$

Note that the expected charging time $\left(\delta_{e v}^{c h a}\right)$ can be calculated easily at EV side before reporting to the CA, given readily available context such as location as well as remain energy level, etc.

2) Estimating Charging Finish Time: At each FCS, by taking into account the on-going charging queue $\left(N_{c}\right)$, the finish time for each MPU of current charging process can be determined by executing Alg. 1, with regard to a list of idle time slots when mobile chargers not occupied. If not all MPUs occupied (line 5 in Alg. 1), current network time $\left(t_{c u r}\right)$ is estimated as the finish time for each MPU.

Specifically, the charging time duration $\left(\delta_{e v}^{c h a}\right)$ to fully replenish the energy of each vehicle $e v \_i$ in the charging queue $\left(\forall i \in N_{c}\right)$ is monitored. Along with the time duration $\left(t_{c u r}-t_{e v_{i} i}^{\text {park }}\right)$ since when the EV is parked, the parking tolerance $\left(\bar{T}_{i}\right)$ of the vehicle is checked by considering the condition $\left(\left(t_{c u r}-t_{e v_{-} i}^{\text {park }}+\delta_{e v_{-} i}^{c h a}\right) \leq T_{i}\right)$ (line 10).
- If the condition is met (line 12), it implies that the vehicle $e v \_i$ can be fully charged before departure. And the finish time is thus given by $\left(t_{c u r}+\delta_{e v_{-} i}^{c h a}\right)$.

- Otherwise (line 14), the EV $e v \_i$ has to terminate the charging process when it hits the departure deadline. In this case, the finish time ends up as $\left(t_{e v_{-} i}^{p} a r k+T_{i}\right)$ instead.

By considering all charging EVs in the queue $N_{c}$, the charging finish time slots associated with MPU identification for future usage are recorded in a map, i.e., IDLE_MAP(mpu_id,avai_time $)$. Since values in $I D L E \_M A P$ are taken to the list $I D L E \_L I S T$ and sorted in an ascending order (line 17), the head value from IDLE_LIST implies the earliest finish time. By executing Alg. 1 at each FCS upon enquiries sent from the CA, charging finish times of all MPUs can be obtained.

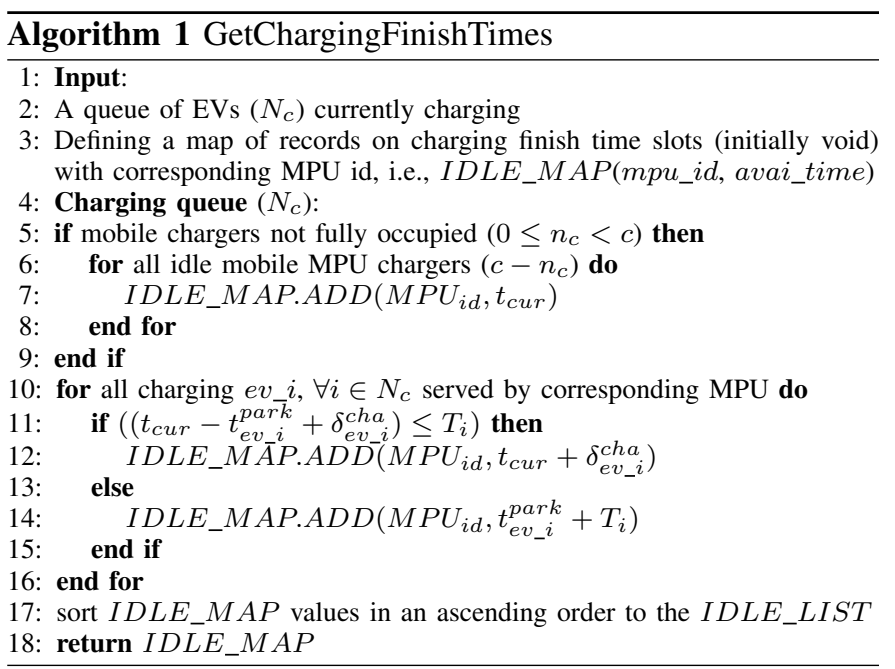

Next, by jointly considering EV reservations $\left(N_{r}\right)$ at each MPU, the $I D L E \_M A P$ can be updated. Further, the waiting time $\left(T_{w}\right)$ can be obtained for the requesting vehicle $e v_{r}$, during which time the candidate MPU would finish all its charging reservations prior to the requesting vehicles reserved time $\left(t_{e v_{r}}^{r e s}\right)$. As such, the value of $\left(T_{w}\right)$ also corresponds to the reservation-queueing duration for the requesting vehicle.

3) Estimating Reservation-Queueing Duration $\left(T_{w}\right)$ : Following Alg. 1, individual MPUs' idle charging times can thus be obtained (IDLE_MAP). However, reservations prior to the requestors $\left(e v \_r\right)$ reserved time (i.e., $\left.t_{e v-r}^{r e s}\right)$ have to be served before heading to the requesting vehicle. As such, idle charging times recorded from the $I D L E \_M A P$ need to be updated for a future moment. In order to achieve this, the heterogeneity of reservations is taken into account, in terms of various reservation time slots that are made earlier than current requesting vehicle $e v_{-} r$, i.e., $\left\{t_{e v_{-} i}^{r e s} \mid i=1, \ldots, n_{r}, t_{e v_{-} i}^{r e s}<\right.$ $\left.t_{e v_{-} r}^{r e s}\right\}$, which are sorted based on specific service discipline, e.g., first reserve first served (FRFS). They are considered so as to predict available charging times for a future moment.

Specifically, the available time for each MPU from $I D L E \_M A P$ is compared with every reserved time $\left\{t_{e v_{i} i}^{r e{ }_{i}} \mid i=1, \ldots, n_{r}, t_{e v_{i} i}^{r e s}<t_{e v_{-} r}^{r e s}\right\}$ within the MPU's reservation queue $\left(N_{r}\right)$ (line 7$)$. In particular, the travel time $\left(T_{t r a}^{i}\right)$ 
for the MPU to take to the reserved vehicle $\left(e v_{-} i\right)$ is also considered.

- If the mobile charger $M P U_{i d}$ arrives late at the reserved EV spot (line 7), i.e., $\left\{I D L E \_M A P . G e t V a l u e\left(M P U_{-} i d\right)+T_{t r a}^{i}>t_{e v_{-} i}^{r e s}\right\}$, only EVs that still need charging service (line 8 to line 12) will be considered for further calculations.

- With concerns on parking deadline, the newly available charging time can be obtained as $t_{\text {new_idle, }}$ by accounting for the condition $\left\{I D L E \_M A P . G e t V a l u e\left(M P U_{-} i d\right)+T_{t r a}^{i}-t_{e v \_i}^{r e s}+\right.$ $\left.\left.\delta_{e v_{-} i}^{c h a}\right) \leq T_{i}\right\}$ (line 9)

- In cases when $M P U_{i d}$ arrives early at the reserved EV spot (line 15), only charging duration needs to be compared with the parking tolerance (line 16). Similarly, $t_{n e w \_i d l e}$ can thus be derived in this case.

Therefore, by iteratively updating the value of $I D L E \_M A P$ (line 22 in Alg. 2), the prediction for a newly idle charging time at the MPU is achieved, thereby enabling the estimation of the expected waiting time. Namely, the requesting vehicle $e v_{r}$ has to wait for additional duration of $T_{w}=t_{\text {new idle }}-t_{\text {old_idle }}$ (line 26) when there are other reservations made earlier. The value of $T_{w}$ is then added to the $W A I T \_M A P$.

By repeating process from line 3 to line 30 in Alg. 2, all MPUs belonging to the same FCS can derive their specific values of $T_{w}$. This way, $W A I T \_M A P$ contains records of $\left(M P U_{i d}, T_{w}\right)$ for all MPUs of an FCS. The list $W A I T_{-} L I S T$ includes all values from $W A I T_{-} M A P$ and is sorted in an ascending order, and thus the head value from $W A I T \_L I S T$ implies the shortest waiting time, which will be adopted later to approximate the expected charging cost value for each FCS $\left(C_{e v}^{f c s}\right)$ with respect to the requesting EV.

4) Global MPU Selection Decision-Making: Upon receiving a recharging demand from the EV $e v \_r$, the CA will request and aggregate context results (i.e., $W A I T \_M A P$, etc.) from all FCSs, which would perform Alg. 1 and Alg. 2 locally. The CA then intelligently enables the global MPU selection decision-making by running Alg. 3. More specifically,

- For the context retrieved from each FCS, get the value of $T_{w}$ from WAIT_LIST.GET(0) (line 3 in Alg. 3), which corresponds to the shortest waiting time. The associated mobile charger $M P U_{i d}$ can be also obtained from $W A I T_{\_} M A P . k e y\left(T_{w}\right)$ (line 4).

- The CA can then approximate the travel time $\left(T_{t r a}\right)$ for the mobile charger $\left(M P U_{i d}\right)$ to take from current location to the requesting EV parking spot (line 5).

- The expected charging cost value $\left(C_{e v_{-} r}^{f c s}\right)$ with associated MPU belonging to each FCS can thus be derived, by accounting for the requestor EVs parking tolerance duration $T_{r}$ (line 6 to line 9).

- With awareness of the cost values of $\left(C_{e v_{r} r}^{f c s}\right)$ from all FCSs (line 11), the optimal MPU ( $M P U_{i d}^{m i n}$ ) with the minimum value is selected (line 13).

Note that the cost value is a joint concern on a range of critical context, regarding estimated waiting time, travel distance as well as expected charging duration. As such, the
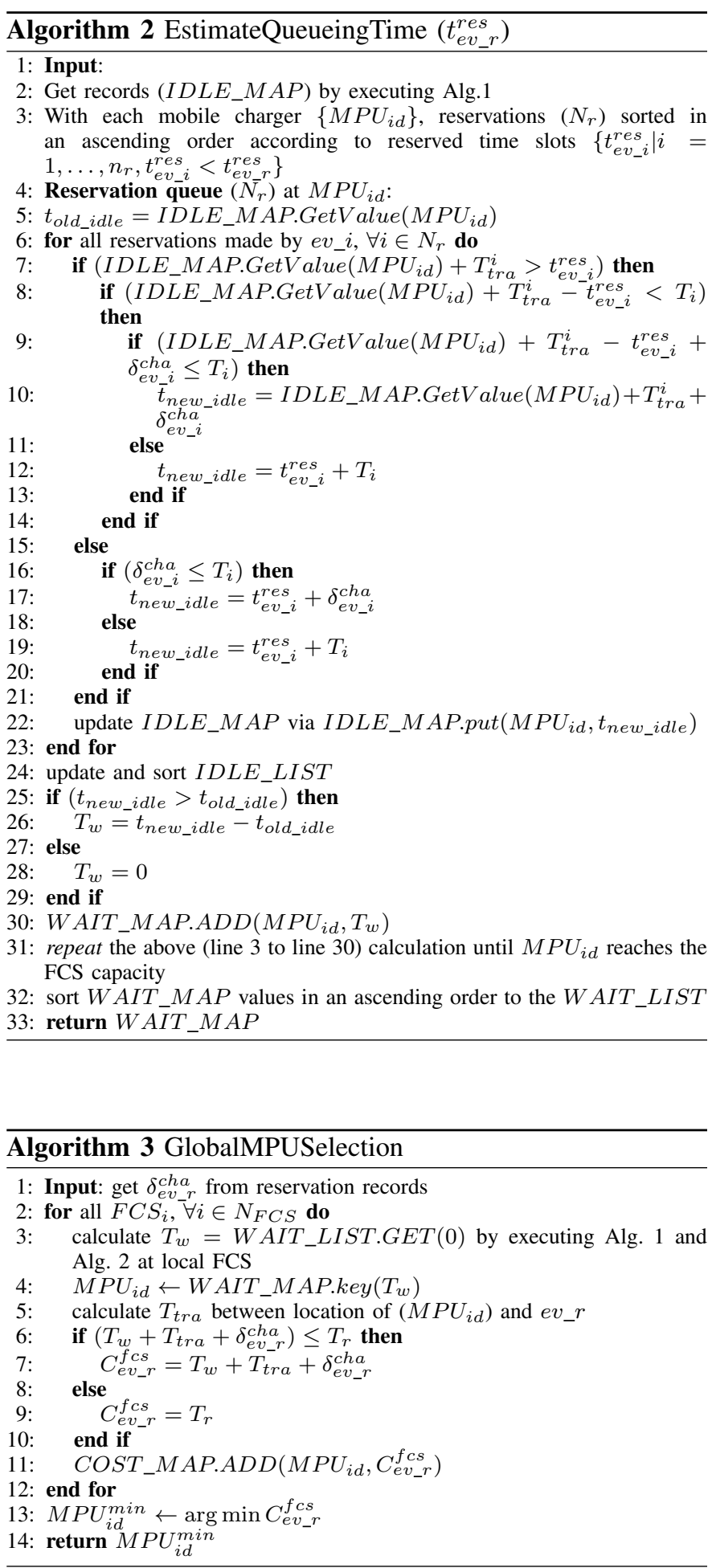

proposed mobile charging solution allows for a rough charging demand balancing among all MPUs, which implies to serve more EVs. Since the travel time is one concern as well when selecting proper MPUs, the candidate MPU tries to reach the demanding EV in a short time. However, such concern does not necessarily guarantee a very low latency, due to the joint concern on various context. Such discussion will be further evaluated in the simulation experimental section. 


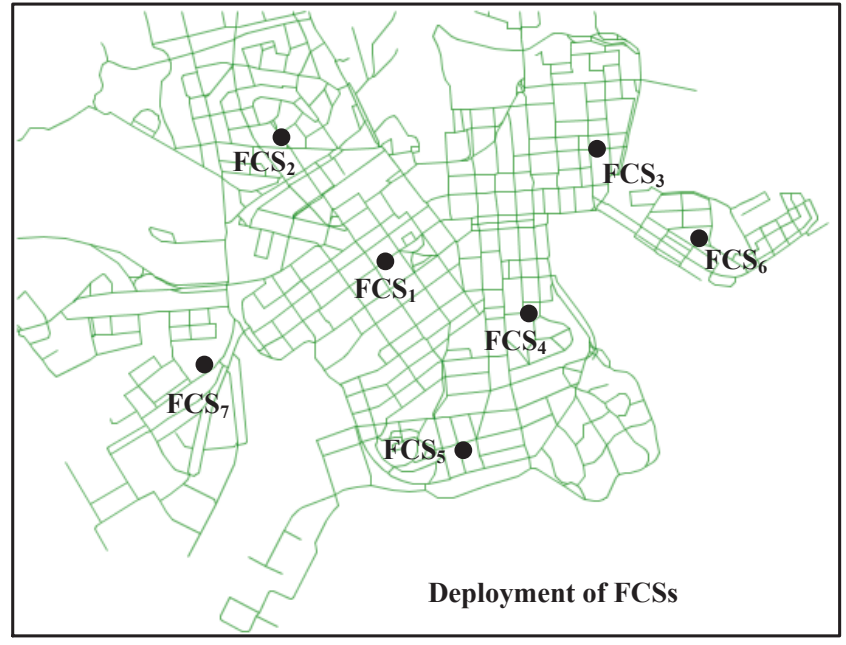

Fig. 5. Simulation scenario of Helsinki city

\section{Simulation Evaluations}

We have built up an entire mobile EV charging system in Opportunistic Network Environment (ONE) [31]. The network scenario is a $4500 * 3400 \mathrm{~m}^{2}$ Helsinki downtown city in Finland as shown in Fig. 5 abstracted from Google map, and the simulation time is set to 12 hours. A total of $246 \mathrm{EVs}$ and 30 mobile chargers (MPUs) are initialized in the network with $[30 \sim 50] \mathrm{km} / h$ variable moving speed. Both MPUs and EVs are configured by following the Hyundai BlueOn specifications in [32], with SOC threshold set as [25\% 55\%]. The fast charging rate of $62 \mathrm{~kW}$ is adopted by both FCSs and MPUs. Besides, 7 fixed charging stations (FCSs) with 5 charging slot each and suffice energy supply are provided through the entire simulation. Each FCS manages a roughly equal amount of MPUs through the simulations.

Destinations of EVs are randomly chosen from locations in the map. And a new destination is selected once the EV reaches the pre-chosen destination. The procedure repeated until the vehicle approaches its battery SOC threshold. Upon this case, a parking spot is randomly selected, with a parking tolerance deadline applied, ranging within $[10 \sim 60]$ min [27]. Upon a charging request, the candidate MPU would travel through the shortest path toward the EVs parking location. While moving or serving EVs, MPUs could hit their SOC threshold. In either cases, MPUs will directly drive to the spatially closest FCS to replenish their energy supply, and any charging processes would be terminated.

The proposed reservation-based mobile charging solution with minimum charging cost value (R-COST) is compared with two other relative schemes, the shortest distance-based MPU selection (DIST) and the earliest available-time MPU selection based on Alg. 1 (AVAIL), both with reservation concerns as well. Note that the two solutions (DIST and AVAIL) are not capable to provide estimations on charging status at MPUs of any future events. And we are interested in the following performance metrics for evaluation.

- Average Charging Time: The average period between the arrival of an MPU and the charging finish time (or fully charged time). It is a metric coupling with EV quality of experience (QoE). A small value implies that MPUs favor to serve EVs with short charging demand and thus, more EVs will be charged.

- Number of Fully Charged EVs: The total amount of EVs that have been fully charged in the network. The metric is coupled with MPU service capability.

- Latency: The time taken from when the EV makes its charging reservation to the time the selected MPU arrives. And an average value is taken. The metric can be kept low if the travel distance for the MPU to reach the requesting $\mathrm{EV}$ is considered.

In the following evaluations, we first analyze the above performance metrics with the assumption of sufficient energy supply provided by all MPUs, so as to focus on varied factors that may play a role in the performance gains. And the assumption will then be further relaxed for more realistic experiments.

\section{A. Impact of Number of Mobile Chargers}

Here, we increase the number of mobile chargers (MPUs) to see how network performances will be affected. As observed from Fig. 6(a), the average charging time slightly decreases under all charging schemes with the increment on the number of MPUs, while the proposed solution R-COST outperforms all other schemes. By contrast, both DIST and AVAIL schemes suffer from longer average charging time period, due to the lack of intelligence on the awareness of critical context, with respect to varied EVs charging duration. As noticed, the AVAIL solution performs slightly better than the DIST, this is mainly due to concerns on on-going charging sessions under AVAIL, which help some level of gains on performance. Results from Fig. 6(a) indicate that the number of MPUs has little impact on the average charging time performance, which is in accordance with our previous analytical results. And we will show in latter analysis that it is mainly the MPU charging power that governs the duration of charging.

As for the impact on the number of fully charged EVs, the influence is significant, as shown in Fig. 6(b). We can see that the number is increased with more MPUs deployed. In particular, the proposed mobile charging scheme R-COST achieves the best performance, by a significant improvement on the number of charged EVs. This is mainly owing to the joint concern on various factors relating to distance as well as charging time and reservation-queueing duration. Also, large number of fully charged EVs are also reached under the DIST solution. The rational is that the possibility can be high under the DIST scheme, when EVs in spatial proximity reserves the same nearby candidate MPU. As such, the MPU is able to serve quite an amount of charging demand within a time period, without having to travel too far. In comparison, the improvement is slight under the AVAIL scheme, due to outdated available times at individual MPUs merely based on Alg. 1.

In Fig. 6(c), it can be seen that the latency is declining with the increment on the number of MPUs. Particularly, there is a significant gain in performance under the proposed solution $\mathrm{R}$-COST as well as the DIST scheme. As observed, the DIST 
solution achieves even less latency when the amount of MPUs is small (under 30), and the performance gain is similar under R-COST and DIST when more MPUs are deployed (more than 60). Since under a distance-based MPU selection scheme, an MPU may find a recharge request that is near to its current location, as discussed previously. And thus a small latency could be achieved. The trend of Fig. 4(c) also accurately conforms to the analytical results as derived previously in Fig. 2 .

\section{B. Impact of Parking Tolerance Deadline}

Here, we analyze how the varied parking deadline would have impact on the concerned performance metrics. As observed from Fig. 7(a), the average charging duration is increased slightly under DIST and AVAIL solutions when the deadline is set long-due, while the results under the proposed R-COST scheme stays the shortest and keeps relatively stable. With a big deadline value, EVs tend to park with longer period and thus, more EVs will wait for charging services. Since EVs are typically with diverse charging duration demand, the chances tend to be high for serving EVs with longer charging time requirement, especially when candidate MPUs are selected regardless of such diversity over varied charging time demand.

As shown in Fig. 7(b), the number of fully charged EVs keeps grow when the parking deadline is set bigger, due to more EVs would stay parked waiting for charging. Not surprisingly, the proposed R-COST solution achieves the best performance. The fact is that with a long-due deadline, MPUs are able to serve more EVs in full charge, and thus the overall performance will be improved. Similarly from previous results, the DIST solution also achieves desirable performance gains, while the AVAIL scheme has the worst.

As for the latency as analyzed in Fig. 7(c), both the proposed R-COST and DIST solutions achieve significant gains similarly to previous analysis, while the AVAIL suffers from very high latency. Note that when EVs tend to park longer, MPUs have to stay longer for charging before heading to the next spot. As a result, the latency is increased a bit when the parking deadline is bigger than $1800 \mathrm{~s}$ in the figure.

\section{Impact of Charging Power}

With higher charging power implemented at MPUs, even shorter average charging time can be achieved under all mobile charging solutions, as shown in Fig. 8(a). As observed, the proposed R-COST outperforms other solutions significantly. Similarly as analyzed above, the DIST solution has the worst performance, while AVAIL achieves relatively better performance gains. We can see from Fig. 8(a) that the MPU charging power has considerable influence on the average charging time.

We can also see great improvement in Fig. 8(b) on the number of fully charged EVs when charging power is enhanced, with the proposed mobile charging solution achieve the best performance. The latency is also improved with high MPU charging power, as shown in Fig. 8(c). The proposed R-COST scheme has moderate higher latency than the DIST scheme when charging power is low, and the gains are improved when MPU acquires a higher charging rate. As noticed, the latency keeps relatively stable under the AVAIL scheme with the increment of MPU charging power. This is mainly due to the lack knowledge of future charging demand under AVAIL charging service, as analyzed in Alg. 1.

The observations from Fig. 8 are of vital importance for practical implementation that, MPUs with high charging power are able to achieve great performance gains, which can be enhanced further by adopting intelligent charging service.

\section{Impact of Charging Capacity}

Here we provide more realistic performance evaluations by relaxing the restriction above that MPUs have suffice energy supply. In the following analysis, MPUs are with limited energy capacity and thus, the power replenishment is needed once MPU SOC thresholds are reached. And a total number of 30 MPUs are deployed. In such cases, MPUs have to drive to the nearest FCS to recharge their battery, and any on-going charging sessions with EVs would be terminated by the MPUs. As such, system performance would be affected as compared to previous analysis.

As shown in Fig. 9(a), with a larger MPU charging capacity, average charging time tend to stay relatively stable under all schemes, while the proposed R-COST achieves the shortest. Similarly to previous analysis, the DIST incurs longer average charging time, due to its unawareness to the heterogeneity of EVs charging durations.

Since MPUs have to get reloaded at FCSs more frequently especially when their capacity is small. In such cases, chances thus become high when MPUs terminate on-going charging sessions. As a result, we can see from Fig. 9(b) that fewer EVs will be fully charged, as compared to the case when MPUs are equipped with suffice power capacity as shown in previous analysis. As shown in the figure, R-COST still achieves the best performance, as compared to DIST and AVAIL.

As shown in Fig. 9(c), the latency stays relatively stable with the increment of MPU charging power capacity. While the DIST scheme achieves desirable performance, the proposed RCOST also achieves great performance gains, in comparison to the AVAIL scheme that suffers from long latency. While MPUs with large power capacity can stay out for long time and charge more EVs, their refill durations tend to be long as well at FCSs. On the other hand, small power MPUs can be recharged in short times at FCSs, however, more frequent returns to FCSs have to be made. As such, the latency at EV side tends to be hardly affected with varied power capacity at MPUs.

From the above observations, we can see that it is not easy to achieve desirable performance gains for all concerned metrics simply under one single scheme. However, an overall system performance improvement can be achieved if a careful design on mobile charging management can be applied, in terms of guaranteed QoE for EV users along with system efficiency for service providers as well. In particular, when MPUs are equipped with suffice energy supply, performance gains can be greatly enhanced as compared to limited MPU power capacity. In fact, the latency would not be a particular concern for EV 


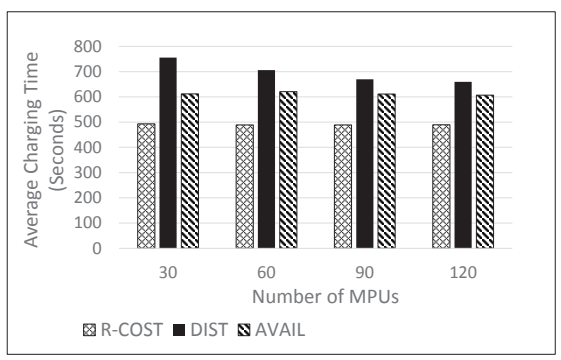

(a) Average Charging Time

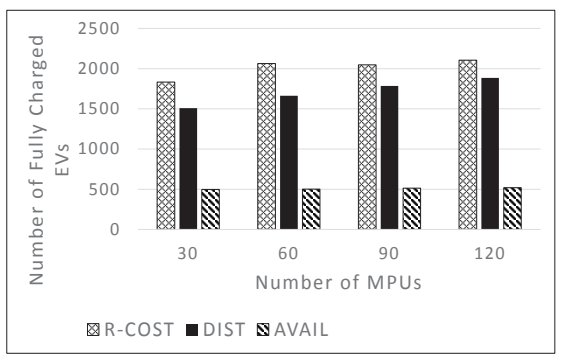

(b) Number of Fully Charged EVs

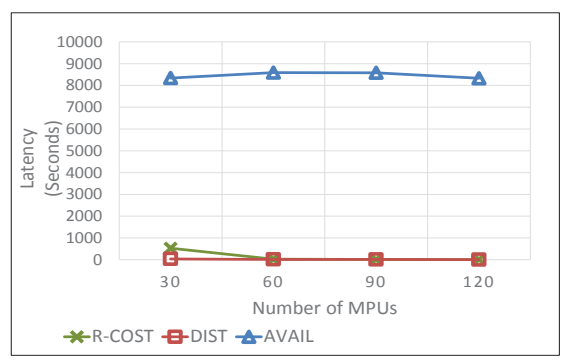

(c) Latency

Fig. 6. Impact of Charging Capacity

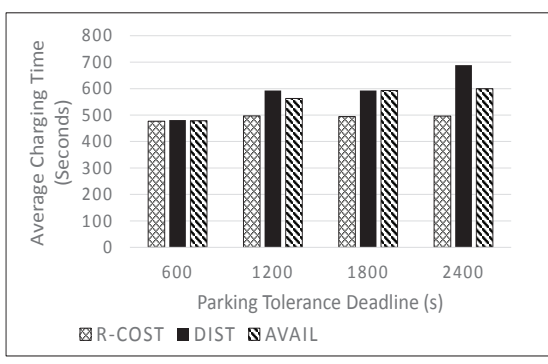

(a) Average Charging Time

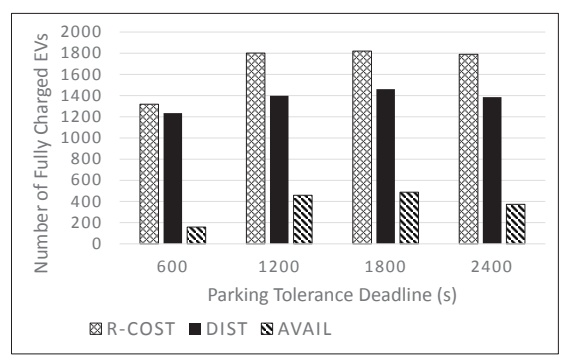

(b) Number of Fully Charged EVs

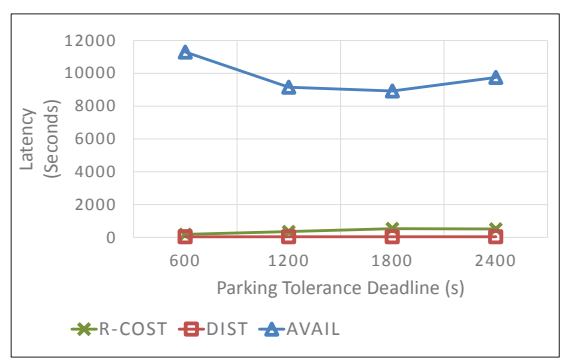

(c) Latency

Fig. 7. Impact of Parking Tolerance Deadline

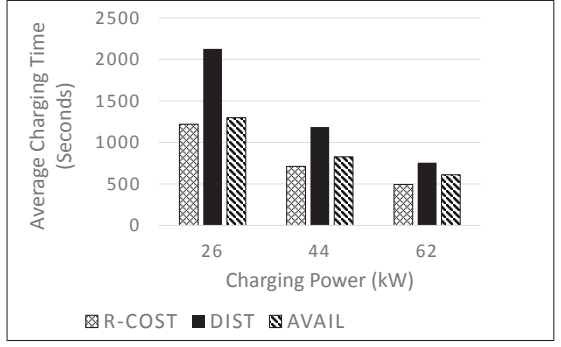

(a) Average Charging Time

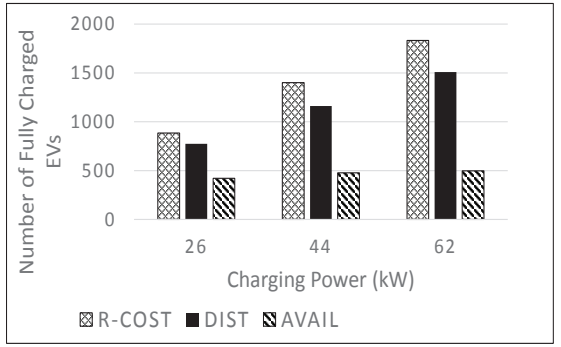

(b) Number of Fully Charged EVs

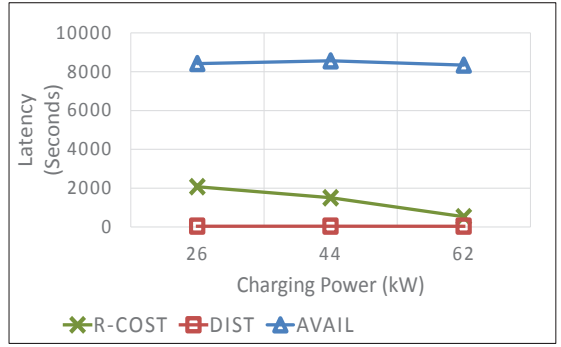

(c) Latency

Fig. 8. Impact of MPU Charging Power

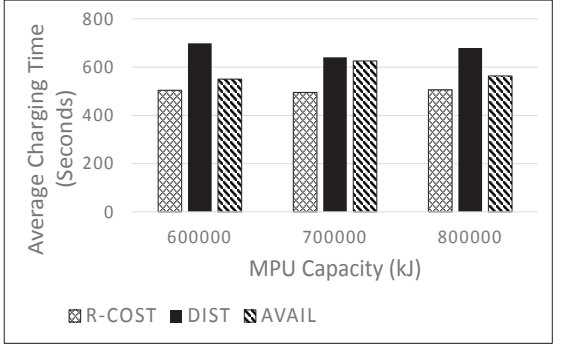

(a) Average Charging Time

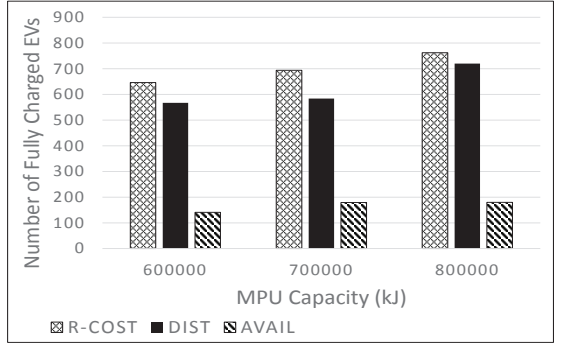

(b) Number of Fully Charged EVs

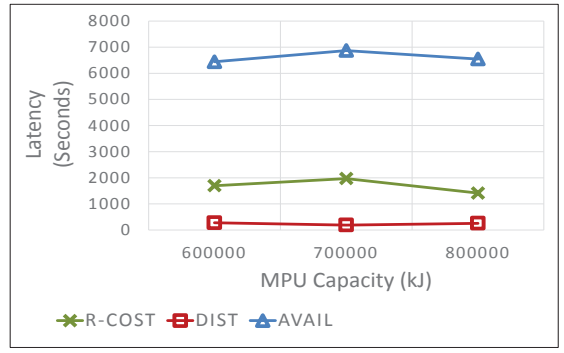

(c) Latency

Fig. 9. Impact of MPU Charging Capacity 
users in most cases, since that EV drivers who order on-site mobile charging services tend to be parked in a relatively long time, e.g., at work places while working for hours [23]. In addition, although by deploying more MPUs would improve the performance, the gains are not very impressive as compared to the upgrade on the MPU charging power.

\section{CONCLUSION}

This paper has present a novel approach to intelligently scheduling mobile charging services toward demanded EVs. Instead of existing systems solely focusing on service discipline on EV selections, this work also considers the scheduling of mobile chargers. By taking into account updated context collected from across the charging network, readily available mobile chargers are predictable. Therefore, EVs with additional charging demand are able to receive prompt services on parking sites by simply making reservations. In particular, candidate mobile chargers could be selected with intelligence by enabling estimations on the available time for a future moment at specific mobile charging units. In order to evaluate critical performance attributes, such as service delay, a theoretical study has been conducted. In order to verify insights observed from the theoretical analysis, a realistic simulation environment has been built up and extensive simulation experiments have been carried out. As observed from the results, the proposed design can significantly enhance the system performance, in terms of the number of fully charged EVs as well as average charging duration, etc. Particularly, with mobile chargers equipped with suffice power capacity, the service delay could be greatly deduced under the proposed solution. By promoting mobile charging services enhanced with mobile charger-selection intelligence, $\mathrm{EV}$ drivers range anxiety as well as charging load over the power grid could be greatly alleviated.

This work considers the scenario where EV charging demand is only served by MPUs. In our future work, a more complex scenario will be considered, wherein EV charging also happens at FCSs, apart from mobile charging services. In such case, when to charge at FCSs/MPUs will be a key issue. Also, EVs and MPUs mobility uncertainty will be taken into account, concerning dynamic traffic conditions, e.g., traffic jams.

\section{REFERENCES}

[1] IEA Global EV Outlook 2019. s://www.iea.org/publications/reports/globalevoutlook2019/

[2] Teslas superchargers. https://www.tesla.com/supercharger

[3] Nissan LEAF Electric Car. https://www.nissanusa.com/electric-cars/leaf/

[4] Kettles D., Electric vehicle charging technology analysis and standards. The Electric Vehicle Transportation Center (EVTC), University of Central Florida, Tech. Rep. FSEC-CR-2057-17, 2017.

[5] Adegbohun, F. et al.,Autonomous Battery Swapping System and Methodologies of Electric Vehicles. Energies, vol. 12, no. 4, 2019.

[6] Aravinthan, V. et al., Controlled electric vehicle charging for mitigating impacts on distribution assets. IEEE Trans. Smart Grid, vol. 6, no. 2, 2015.

[7] Cao, Yue et al. Toward efficient, scalable, and coordinated on-the-move EV charging management. IEEE Wireless Communications, vol. 24, no. $2,2017$.

[8] Mukherjee, J. C. et al., A review of charge scheduling of electric vehicles in smart grid. IEEE Systems Journal, vol. 9, no. 4, 2014.
[9] IS Bayram et al., Unsplittable load balancing in a network of charging stations under QoS guarantees. IEEE Trans. Smart Grid. vol. 6, no. 2014.

[10] Ovalle, A. et al., Optimal management and integration of electric vehicles to the grid: Dynamic programming and game theory approach. in Proc. IEEE International Conference on Industrial Technology (ICIT), 2015.

[11] Luo, C. et al., Stochastic dynamic pricing for EV charging stations with renewable integration and energy storage. IEEE Trans. Smart Grid, vol. 9, no. 2, 2017.

[12] SAE Vehicle electrictification standards. http://www.sae.org/smartgrid/

[13] Un-Noor, Fuad et al., A comprehensive study of key electric vehicle (EV) components, technologies, challenges, impacts, and future direction of development. Energies, vol. 10, no. 8, 2017.

[14] Awasthi, A. et al., Optimal planning of electric vehicle charging station at the distribution system using hybrid optimization algorithm. Energy, vol. 133, no. 1, 2017.

[15] K Gowri et al., Vehicle to grid communication standards development, testing and validation: status report. Pacific Northwest National Laboratory, Technical Report, No. PNNL-20844, 2011.

[16] Tang, D. et al., Probabilistic modeling of nodal charging demand based on spatial-temporal dynamics of moving electric vehicles. IEEE Trans. Smart Grid, vol. 7, no. 2, 2015.

[17] Cao, Yue et al., A cost-efficient communication framework for batteryswitch-based electric vehicle charging. IEEE Communications Magazine, vol. 55, no. 5, 2017.

[18] Luo, Y. et al., Optimal charging scheduling for large-scale EV (electric vehicle) deployment based on the interaction of the smart-grid and intelligent-transport systems. Energy, vol. 97, no. 1, 2016.

[19] Rigas, Emmanouil S. et al., Managing electric vehicles in the smart grid using artificial intelligence: A survey. IEEE Trans. Intelligent Transportation Systems,vol. 16, no. 4, 2015.

[20] N. Ghiasnezhad Omran et al., Location-based forecasting of vehicular charging load on the distribution system. IEEE Trans. Smart Grid, vol. 5, no. 2, 2014.

[21] Wang, D. et al., Modeling of plug-in electric vehicle travel patterns and charging load based on trip chain generation. Journal of Power Sources, vol. 359 , no. 1, 2017.

[22] M Wang et al., Spatio-temporal coordinated v2v energy swapping strategy for mobile pevs. IEEE Trans. Smart Grid, vol. 9, no. 3, 2016.

[23] Huang, Shisheng et al., Design of a mobile charging service for electric vehicles in an urban environment. IEEE Trans. Intelligent Transportation Systems, vol. 16, no. 2, 2015.

[24] D. Gross et al., Fundamentals of Queueing Theory. Hoboken, NJ, USA: Wiley, 2013.

[25] Freewire Technologies, https://www.freewiretech.com/mobile-power/

[26] Philip, Johan, The probability distribution of the distance between two random points in a box. KTH mathematics, Royal Institute of Technology, 2007.

[27] Cao, Yue et al., An EV charging management system concerning drivers trip duration and mobility uncertainty. IEEE Trans. Systems, Man, and Cybernetics: Systems, vol. 48, no. 4, 2018.

[28] MOBI CHARGER SERIES. https://www.freewiretech.com/ev-charging/

[29] Chevrolet Volt. http://www.chevrolet.ca/volt-electric-car.html

[30] S. M. Ross, Stochastic Processes. John \& Wiley Sons, 1996.

[31] Keränen et al, The ONE simulator for DTN protocol evaluation. Proceedings of the 2nd international conference on simulation tools and techniques, 2009.

[32] Hundai BlueOn. en.wikipedia.org/wiki/Hyundai BlueOn

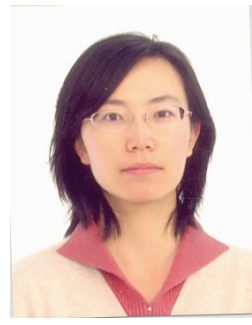

Xu Zhang received the B.Eng. degree in telecommunication engineering from the Xidian University, Xi'an, China, in 2007, and the M.Sc. and Ph.D degrees from the University of Science and Technology, Beijing, China, and the University of Surrey, Surrey, U.K., in 2010 and 2015, respectively. She is currently a Lecturer with the Department of Computer Science, Xi'an University of Technology, Xi'an, China. Her research interests include networking and network management, including aspects such networking and E-mobility. 


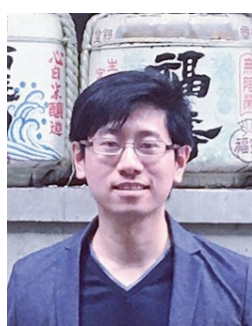

Yue Cao received the $\mathrm{PhD}$ degree from the Institute for Communication Systems (ICS), at University of Surrey, Guildford, UK in 2013. He is currently the Professor at School of Transportation Science and Engineering, Beihang University, China. His research interests focus on Intelligent Transport Systems. He is the Associate Editor of IEEE Access, Springer EURASIP Journal on Wireless Communications and Networking, KSII Transactions on Internet and Information Systems etc.

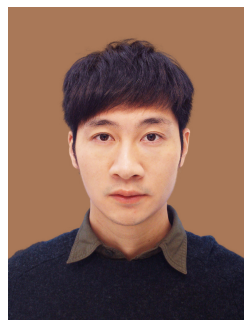

Linyu Peng received his Ph.D. degree in mathematics from University of Surrey, Guildford, U.K., in 2013. From 2013 to 2020, he worked at Waseda University, Tokyo, Japan. He is currently an Assistant Professor at the Department of Mechanical Engineering, Keio University, Tokyo, Japan. His research interests concern on pure and applied mathematics, including mathematical optimisation, algebraic and geometric theories of differential equations and finite difference equations, and information geometry.

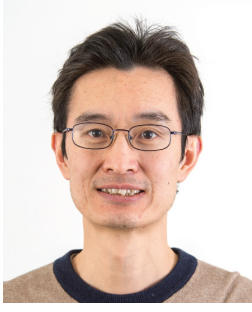

Jichun Li received his Ph.D. degree in mechanical engineering from King's College London, University of London, U.K. in 2013. He is currently a Lecturer with the School of Computer Science and Electronic Engineering, University of Essex, U.K. His main research interests include neural networks, robotics, intelligent transportation and communication, control, IoT and AI solutions for bespoke automated systems for chemical, medical, energy, agri-food and biosciences industries. He is a member of the IEEE, IET, and IMeChE.

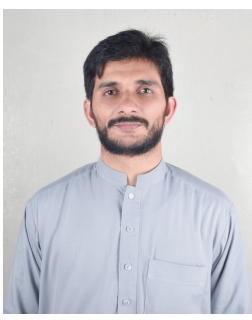

Naveed Ahmad received his BSc (Computer Sciences) honors degree from University of Peshawar, Pakistan in 2007, and PhD in Electronics and Electrical Engineering from Institute of Communication System, University of Surrey, UK in 2013. He is currently serving as Assistant Professor in Department of Computer Science, University of Peshawar, Pakistan. He research interests include routing, security and privacy in emerging networks such as Vehicular Adhoc Networks (VANETS), Delay Tolerant Networks (DTN) and Internet of Things (IoT).

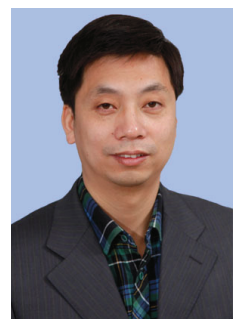

Shengping Yu obtained a bachelor degree from Xi'an University of Architecture and Technology in 1999, a master degree and a doctorate from Northeastern University in 2005 and 2017, respectively. His research interests include dynamic scheduling of complex industrial processes, dynamic scheduling of high-speed railway trains, intelligent analysis and prediction of overall process quality, intelligent optimization, and manufacturing execution systems. He has published over 30 peer reviewed international journal and conference papers, and authorized 7

Chinese invention patents. 